Editorial

\title{
Sjögren's Syndrome: Animal Models, Etiology, Pathogenesis, Clinical Subtypes, and Diagnosis
}

\author{
Long Shen $\mathbb{D}^{1},{ }^{1}$ Jing He $\mathbb{D}^{2},{ }^{2}$ Jill M. Kramer $\mathbb{D}^{1},{ }^{1}$ and Vatinee $Y$. Bunya $\mathbb{D}^{3}$ \\ ${ }^{1}$ University at Buffalo, Buffalo, USA \\ ${ }^{2}$ Peking University People's Hospital, Beijing, China \\ ${ }^{3}$ Scheie Eye Institute, University of Pennsylvania, Philadelphia, USA
}

Correspondence should be addressed to Long Shen; shenl@buffalo.edu

Received 9 May 2019; Accepted 9 May 2019; Published 20 May 2019

Copyright (c) 2019 Long Shen et al. This is an open access article distributed under the Creative Commons Attribution License, which permits unrestricted use, distribution, and reproduction in any medium, provided the original work is properly cited.

Sjögren's syndrome (SS) is a chronic autoimmune disease that classically affects the lacrimal and salivary glands resulting in dry eyes and dry mouth and can affect almost any organ system in the body including the lungs, kidney, and central nervous system [1]. Many systemic aspects of SS are described, including B cell lymphoma in addition to pulmonary and renal pathoses [2-4]. Diagnosis often takes several years once symptoms manifest [5], and even after the diagnosis is rendered, there are currently no treatments available that address the underlying disease etiology.

While the pathogenesis of SS is not well understood, both innate and adaptive immune responses are implicated in disease initiation and progression. In the innate response, an antiviral response is mounted through the recognition of viral nucleic acids by Toll-like receptors (TLRs). This recognition leads to the upregulation of the type 1 interferon (IFN) pathway. However, the means by which immune activation is initiated and maintained remain incompletely understood. Activation of TLRs is critical for the progression of numerous autoimmune diseases [6], although there are relatively few studies regarding the role of these receptors in SS. Studies in mice and humans reveal that TLRs are potent mediators of inflammation in SS. TLRs are expressed and functional in salivary tissue, and TLRs in peripheral blood cells of SS patients are also upregulated and hyperresponsive to ligation. In addition, interferon signatures have been detected in the blood and in the labial salivary gland tissues of patients with pSS [7]. However, it is unclear whether TLRs are activated by microbial products or host-derived ligands in SS [8]. Both animal models and patient studies are instrumental in understanding causes of TLR dysfunction in SS [9]. Studies that identify specific TLRs and clinically relevant ligands will likely lead to the development of novel therapeutics that will diminish chronic inflammation that is a characteristic of SS.

Several viruses have been implicated as possible environmental triggers of SS including Epstein-Barr virus (EBV), cytomegalovirus (CMV), human herpes virus type 8 (HHV8 ), human T-lymphotropic virus type 1 (HTLV-1), hepatitis $\mathrm{C}$ virus, and enterovirus [10]. In addition, paramyxovirus, which causes mumps, may persist in salivary glands and could provide the necessary trigger to initiate autoimmunity pathogenesis of SS in certain genetically susceptible individuals [11]. Cytokines, B cell growth factor, and serum B cellactivating factor (BAFF) levels also play an important role in the pathogenesis of SS. They are altered in patients with SS and correlate with antibodies to Ro and La [12, 13]. Previous studies have shown that most IL-14 $\alpha$ transgenic mice develop gastrointestinal B cell lymphomas [14].

Most often, SS presents with polymorphic clinical presentations including the involvement of various systemic organs, which can result in delays in diagnosis. While SS is typically not associated with significant mortality, patients who demonstrate renal involvement are at risk for lifethreatening complications. Renal involvement in SS is relatively rare and is seen in approximately $5-10 \%$ of SS patients [15-18]. Studies are needed to understand the mechanisms that govern kidney dysfunction in SS and to uncover the optimal diagnostic strategies for SS patients with renal complications. In addition, it is important to identify SS patients who 
are at high risk of renal disease in SS in order to manage these individuals appropriately.

Non-Hodgkin lymphoma is another serious systemic manifestation of SS $[19,20]$. SS is characterized by B cell hyperactivity, and the lymphomas that arise in SS patients originate exclusively from B cells [21]. There are several clinical and serological findings that are associated with lymphoma development in SS patients, including anemia, neutropenia, and thrombocytopenia [22]. In addition, BAFF polymorphisms have been identified, and BAFF levels tend to be elevated in patients who develop lymphoma [12, 23-25]. While the relationship between lymphoma development, BAFF levels, and autoantibodies is not well understood, further work is needed to identify autoantibodies that may be indicative or predictive of lymphoma development in SS [21].

The diagnosis of SS can be very challenging due to the absence of specific clinical manifestations in the early stages of the disease and the lack of noninvasive diagnostic methods with high specificity and sensitivity. This can lead to significant delays in treatment and worse clinical outcomes. Therefore, novel biomarkers and imaging methods to help recognize the disease at an early stage are needed. For example, antibodies to salivary gland protein 1 (SP1), carbonic anhydrase 6 (CA6), and parotid secretory protein autoantibodies (PSP) were first described in a mouse model for SS and have also been found in SS patients together and without anti-SSA/Ro, as well as in patients with idiopathic dry mouth and dry eye disease [26]. However, despite the discovery of new biomarkers such as these, there are continued diagnostic delays due to the limited understanding of the sequence of events that trigger the activation of the autoimmunity against specific antigenic targets. It is important to study and elucidate the pathways leading to the activation of the antigenic targets as it may lead to more precise disease diagnosis and to the development of specific targeted therapies.

The ocular manifestations of SS often cause substantial morbidity as patients experience significant decreases in quality of life and visual functioning as a result of the disease [27]. Approximately $10 \%$ of dry eye patients have underlying SS, but because dry eye is a highly prevalent condition in the general population [27], it is not possible to work-up all dry eye patients for SS. Therefore, better tests that distinguish SS from other causes of dry eye are needed. In addition, further work is required to understand the inciting events that lead to the development of the ocular manifestations of SS. While immune dysfunction is thought to underlie the ocular dysfunction observed, [28] the contribution of environmental insults and genetics to the disease remain incompletely understood [29]. Thus, further work to understand the mechanisms that govern ocular dysfunction and to develop tests for dry eye that identify patients with SS will have a significant impact.

The goal of this special issue is to highlight recent advances in the understanding of the etiopathogenesis, varying clinical presentations, and diagnosis of SS. For example, Z. Mackiewicz et al. in their article entitled "Sjögren's Syndrome: Concerted Triggering of Sicca Conditions" evaluate the role of the paramyxovirus in SS. J. Kramer and J. Kiripolsky in their paper "Current and Emerging Evidence for
Toll-Like Receptor Activation in Sjögren's Syndrome" explore the role of TLRs in the pathogenesis of SS based on findings in the various mouse models. They discuss the role and significance of putative damage-associated molecular patterns in SS. J. Luo et al. discuss renal involvement of SS, its diverse clinical presentation, and the role of renal biomarkers in kidney damage assessment in their article "High-Risk Indicators of Renal Involvement in Primary Sjogren's Syndrome: A Clinical Study of 1002 Cases." Z. Xian and colleagues, in their study entitled "Association between B Cell Growth Factors and Primary Sjögren's SyndromeRelated Autoantibodies in Patients with Non-Hodgkin's Lymphoma," describe the relation between the cytokines BAFF and IL-14 with various traditional autoantibodies (anti-SSA/Ro) and newer tissue-specific autoantibodies. They also describe the role of cytokines and autoantibodies for the stratification of SS patients with gastrointestinal lymphomas. Finally, S. Karakus and colleagues conducted a cross-sectional study of dry eye patients to investigate the clinical significance of anti-salivary gland protein 1 (SP1), anti-carbonic anhydrase 6 (CA6), and anti-parotid secretory protein (PSP) autoantibodies. In their manuscript "Clinical Correlations of Novel Autoantibodies in Patients with Dry Eye," they demonstrate that disease severity stratification may be feasible using new biomarkers and conclude that anti-CA6 is seen in patients with severe aqueous-deficient dry eye. Taken together, it appears that a new era is on the horizon for a better understanding of the clinical manifestations, diagnosis, etiology, and pathogenesis of SS.

\section{Conflicts of Interest}

Drs. Long Shen, Vatinee Bunya, Jing He, and Jill M. Kramer declare that they have no conflicts of interest related to this work. Dr. Bunya receives grant funding from Bausch \& Lomb/Immco Diagnostics.

Long Shen Jing $\mathrm{He}$

Jill M. Kramer

Vatinee Y. Bunya

\section{References}

[1] R. I. Fox, “Sjögren's syndrome," The Lancet, vol. 366, no. 9482, pp. 321-331, 2005.

[2] M. Ramos-Casals, P. Brito-Zerón, R. Seror et al., "Characterization of systemic disease in primary Sjögren's syndrome: EULAR-SS Task Force recommendations for articular, cutaneous, pulmonary and renal involvements," Rheumatology, vol. 54, no. 12, pp. 2230-2238, 2015.

[3] A. S. Malladi, K. E. Sack, S. C. Shiboski et al., "Primary Sjögren's syndrome as a systemic disease: A study of participants enrolled in an International Sjögren's syndrome registry," Arthritis Care \& Research, vol. 64, no. 6, pp. 911-918, 2012.

[4] F. Vivino, V. Y. Bunya, G. Massaro-Giordano et al., "Sjogren's syndrome: an update on disease pathogenesis, clinical manifestations and treatment," Clinical Immunology, vol. 203, pp. 81-121, 2019, Epub 2019/04/26. 
[5] Sjogren's Syndrome Foundation - Diagnosis, “Sjogren's Syndrome Foundation, Inc.," 2018, https://www.sjogrens.org/ home/about-sjogrens/diagnosis.

[6] J. Q. Chen, P. Szodoray, and M. Zeher, "Toll-like receptor pathways in autoimmune diseases," Clinical Reviews in Allergy and Immunology, vol. 50, no. 1, pp. 1-17, 2016.

[7] J. C. Hall, A. N. Baer, A. A. Shah et al., "Molecular subsetting of interferon pathways in Sjögren's syndrome," Arthritis \& Rheumatology, vol. 67, no. 9, pp. 2437-2446, 2015.

[8] J. Kiripolsky, L. G. McCabe, and J. M. Kramer, "Innate immunity in Sjögren's syndrome," Clinical Immunology, vol. 182, pp. 4-13, 2017.

[9] A. B. Peck and C. Q. Nguyen, "What can Sjögren's syndromelike disease in mice contribute to human Sjögren's syndrome?," Clinical Immunology, vol. 182, pp. 14-23, 2017.

[10] P. Sandhya, B. Kurien, D. Danda, and R. Scofield, "Update on pathogenesis of Sjogren's syndrome," Current Rheumatology Reviews, vol. 13, no. 1, pp. 5-22, 2017.

[11] N. Holdgate and E. W. S. Clair, "Recent advances in primary Sjogren's syndrome," F1000Research, vol. 5, 2016.

[12] X. Mariette, S. Roux, J. Zhang et al., "The level of BLyS (BAFF) correlates with the titre of autoantibodies in human Sjögren's syndrome," Annals of the Rheumatic Diseases, vol. 62, no. 2, pp. 168-171, 2003.

[13] L. Shen and L. Suresh, "Autoantibodies, detection methods and panels for diagnosis of Sjögren's syndrome," Clinical Immunology, vol. 182, pp. 24-29, 2017.

[14] L. Shen, C. Zhang, T. Wang et al., "Development of Autoimmunity in IL-14 $\alpha$-Transgenic Mice," The Journal of Immunology, vol. 177, no. 8, pp. 5676-5686, 2006.

[15] R. Evans, A. Zdebik, C. Ciurtin, and S. B. Walsh, "Renal involvement in primary Sjögren's syndrome," Rheumatology, vol. 54, no. 9, pp. 1541-1548, 2015.

[16] H. X. Yang, J. Wang, Y. B. Wen et al., "Renal involvement in primary Sjögren's syndrome: A retrospective study of 103 biopsy-proven cases from a single center in China," International Journal of Rheumatic Diseases, vol. 21, no. 1, pp. 223229, 2018.

[17] M. Jasiek, A. Karras, V. Le Guern et al., "A multicentre study of 95 biopsy-proven cases of renal disease in primary Sjögren's syndrome," Rheumatology, vol. 56, no. 3, pp. 362-370, 2017.

[18] A. V. Goules, I. P. Tatouli, H. M. Moutsopoulos, and A. G. Tzioufas, "Clinically Significant Renal Involvement in Primary Sjögren's Syndrome: Clinical Presentation and Outcome," Arthritis and Rheumatism, vol. 65, no. 11, pp. 2945-2953, 2013.

[19] C. P. Mavragani and H. M. Moutsopoulos, "Sjögren's syndrome," Annual Review of Pathology: Mechanisms of Disease, vol. 9, no. 1, pp. 273-285, 2014.

[20] A. Alunno, M. C. Leone, R. Giacomelli, R. Gerli, and F. Carubbi, "Lymphoma and Lymphomagenesis in Primary Sjögren's Syndrome," Frontiers in Medicine, vol. 5, p. 102, 2018.

[21] A. V. Goules and A. G. Tzioufas, "Lymphomagenesis in Sjögren's syndrome: Predictive biomarkers towards precision medicine," Autoimmunity Reviews, vol. 18, no. 2, pp. 137143, 2019.

[22] A. Papageorgiou, D. C. Ziogas, C. P. Mavragani et al., "Predicting the outcome of Sjogren's syndrome-associated nonHodgkin's lymphoma patients," PLoS One, vol. 10, no. 2, article e0116189, 2015.
[23] M. V. Jonsson, P. Szodoray, S. Jellestad, R. Jonsson, and K. Skarstein, "Association between circulating levels of the novel TNF family members APRIL and BAFF and lymphoid organization in primary Sjögren's syndrome," Journal of Clinical Immunology, vol. 25, no. 3, pp. 189-201, 2005.

[24] A. J. Novak, S. L. Slager, Z. S. Fredericksen et al., "Genetic variation in B-cell-activating factor is associated with an increased risk of developing B-cell non-Hodgkin lymphoma," Cancer Research, vol. 69, no. 10, pp. 4217-4224, 2009.

[25] A. Nezos, A. Papageorgiou, G. Fragoulis et al., "B-Cell activating factor genetic variants in lymphomagenesis associated with primary Sjogren's syndrome," Journal of Autoimmunity, vol. 51, pp. 89-98, 2014.

[26] S. Karakus, A. N. Baer, D. Agrawal, M. Gurakar, R. W. Massof, and E. K. Akpek, "Utility of novel autoantibodies in the diagnosis of Sjögren's syndrome among patients with dry eye," Cornea, vol. 37, no. 4, pp. 405-411, 2018.

[27] E. K. Akpek, V. Y. Bunya, and I. J. Saldanha, “Sjögren's Syndrome," Cornea, vol. 38, no. 5, pp. 658-661, 2019.

[28] J. L. Reyes, D. T. Vannan, B. Eksteen et al., "Innate and adaptive cell populations driving inflammation in dry eye disease," Mediators of Inflammation, vol. 2018, Article ID 2532314, 12 pages, 2018.

[29] G. Galperin, M. Berra, M. I. Marquez, M. Mandaradoni, J. Tau, and A. Berra, "Impact of environmental pollution on the ocular surface of Sjögren's syndrome patients," Arquivos Brasileiros de Oftalmologia, vol. 81, no. 6, pp. 481-489, 2018. 


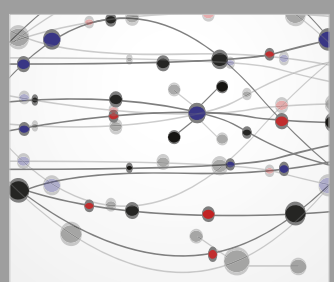

The Scientific World Journal
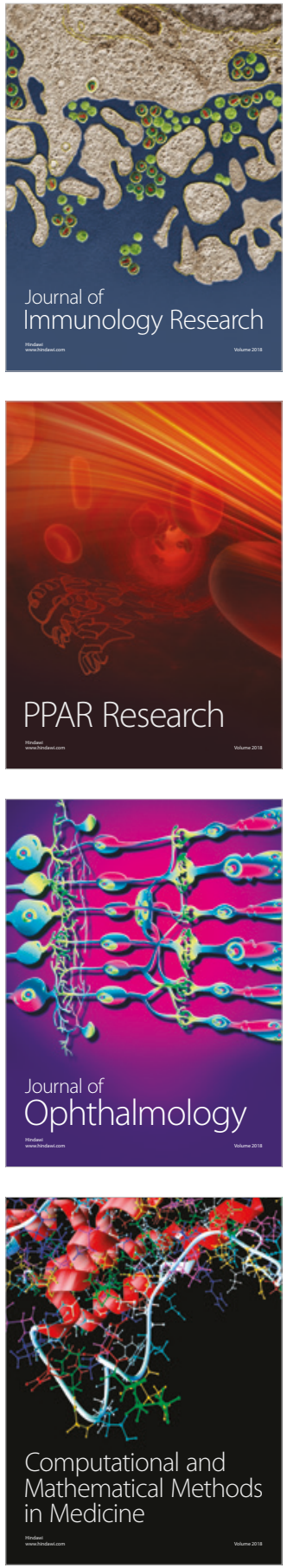

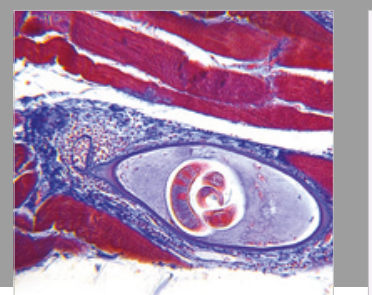

Gastroenterology Research and Practice

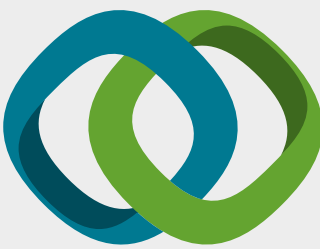

\section{Hindawi}

Submit your manuscripts at

www.hindawi.com
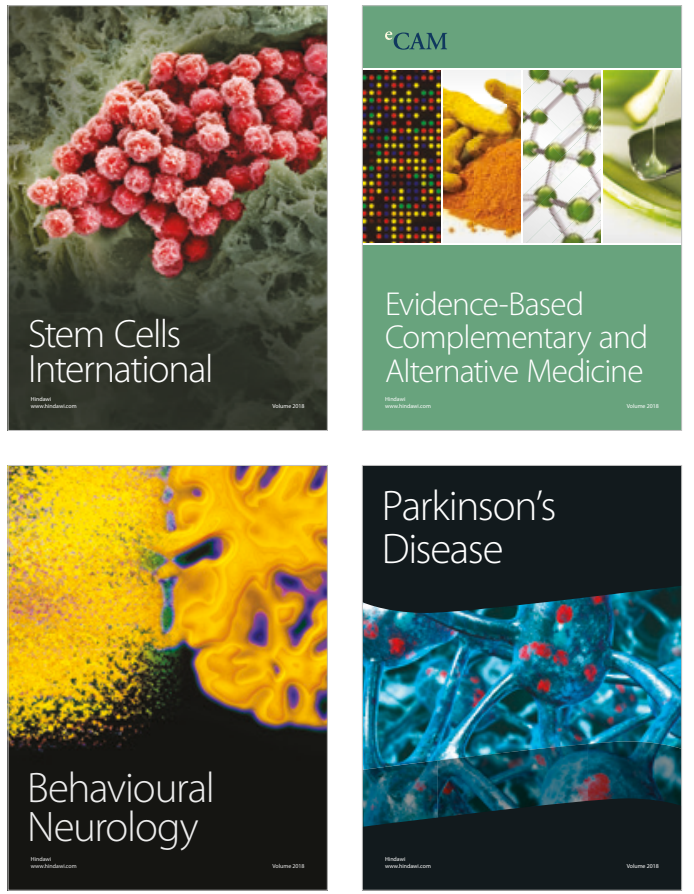

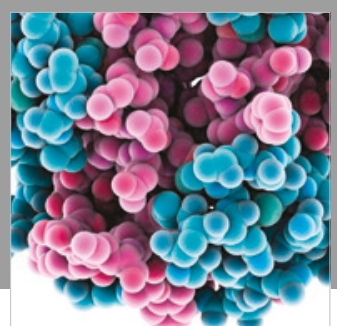

ournal of

Diabetes Research

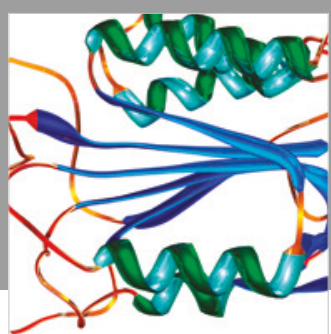

Disease Markers
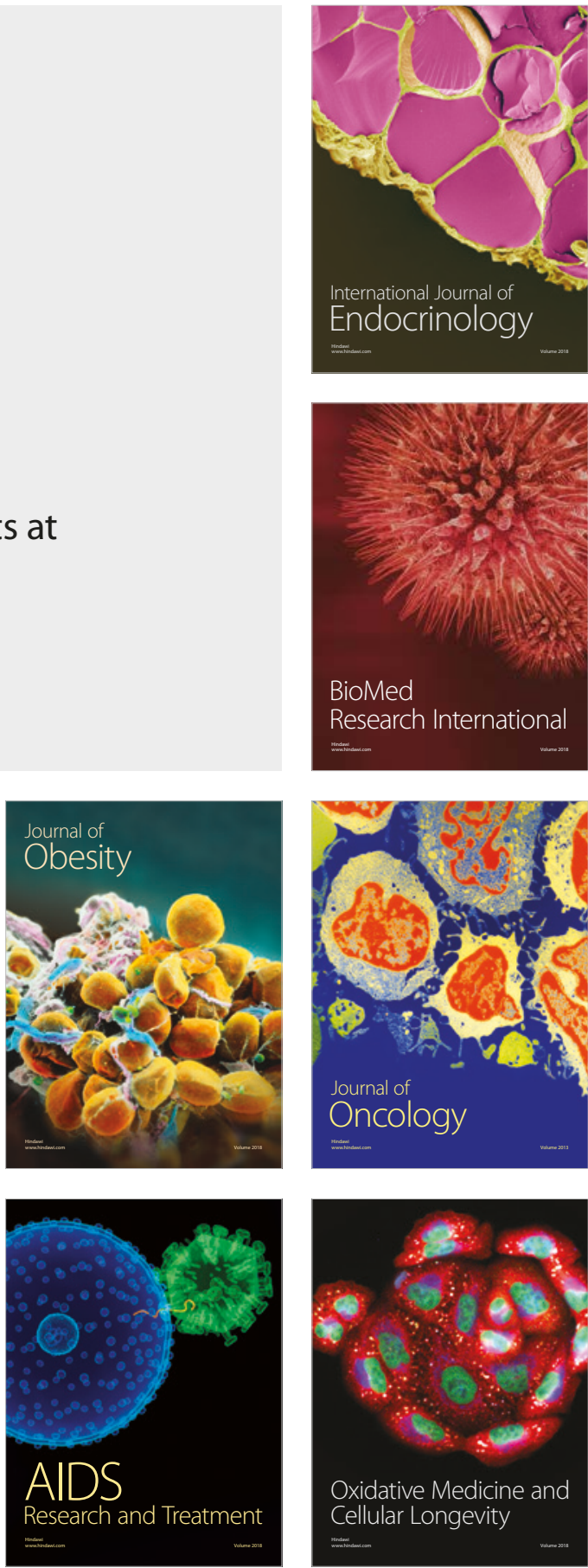